\title{
МОДЕЛИРОВАНИЕ ВКЛЮЧЕНИЯ АКТИВНОГО ПОТРЕБИТЕЛЯ НА ПАРАЛЛЕЛЬНУЮ РАБОТУ С СИСТЕМОЙ ЭЛЕКТРОСНАБЖЕНИЯ НЕФТЕГАЗОДОБЫВАЮЩЕГО ПРЕДПРИЯТИЯ
}

\author{
Косарев Борис Андреевич 1 , \\ BorisK_88@mail.ru
}

Федоров Владимир Кузьмич 1 , el.tech.omgtu@gmail.com

\author{
Хамитов Рустам Нуриманович1,2, \\ apple_27@list.ru \\ 1 Омский государственный технический университет, \\ Россия, 644050, г. Омск, пр. Мира, 11. \\ 2 Тюменский индустриальный университет, \\ Россия, 625000, г. Тюмень, ул. Володарского, 38.
}

\begin{abstract}
Актуальность. В районах добычи нефти и газа в связи с интенсивным развитием распределенной энергетики можно прогнозировать появление активных потребителей, использующих работающие на попутном нефтяном газе газотурбинные установки. При этом представляется недостаточно изученным вопрос переходных процессов, возникающих при подключении активного потребителя к системе электроснабжения нефтегазодобывающего предприятия. При сильных возмущениях режима при условии наличия нелинейных элементов переходные процессы могут завершиться устойчивыми хаотическими колебаниями - аварийным режимом работы электросистемы. Таким образом, существует необходимость изучения подключения активного потребителя к централизованным системам электроснабжения в районах добычи нефрти и газа.

Цель: рассмотрение переходных процессов, возникающих при включении электрических систем активного потребителя и нефтегазодобывающего предприятия на параллельную работу.

объекты: электрические системы активного потребителя и нефтегазодобывающего предприятия.

Методы. Переходные процессы в электрических системах активного потребителя и нефртегазодобывающего предприятия исследуются путем компьютерного моделирования в программном пакете Simscape Power Systems (Matlab). B компьютерной модели при построении структурных схем электросистем, выборе мощности элементов и уровня напряжений использованы основы электроснабжения промышленных предприятий. Расчет значений параметров элементов электросистем выполнен на основе теории электрических машин и теоретических основ электротехники.

Результаты моделирования показывают при включении методом точной синхронизации генератора активного потребителя на параллельную работу с внешней системой нормальный переходньй режим длительностью 4 секунды. При несинхронном включении генератора в электросистеме возникают устойчивые хаотические колебания. На хаотические колебания указывает апериодический характер изменения во времени значений частоты, напряжения и мощности, а также нестранный хаотический аттрактор напряжения с положительным показателем Ляпунова 0,44. При хаотическом режиме работы на шинах промысловой подстанции диапазон изменений значений активной мощности составляет 18 кВт, частоты - 1,5 Ги, напряжения - $400 \mathrm{~B}$.
\end{abstract}

\section{Ключевые слова:}

Распределенная генерация, активный потребитель, хаотические колебания, странный нехаотический аттрактор, показатель Ляпунова, компьютерное моделирование.

\section{Введение}

В настоящее время одним из перспективных направлений развития российской и мировой энергетики является создание систем электроснабжения с распределенной генерацией [1, 2]. Распределенная генерация (РГ) - это производство электроэнергии в непосредственной близости от потребителя, что позволяет минимизировать потери при передаче электроэнергии и эффективно использовать доступные энергоресурсы, в том числе возобновляемые источники энергии [3-5]. При этом возникает понятие «prosumer», или активный потребитель. Активный потребитель - это потребитель, который может производить электроэнергию для собственных нужд и направлять избыток мощности во внешнюю сеть. Взаимодействие активного потребителя и внешней электросистемы является сложным процессом, управлять которым эффективно при помощи виртуальных подстанций или «умной сети» (smart grid) $[6,7]$. Основой «умной сети» являются алгоритмы управления, которые в настоящее время интенсивно исследуются и совершенствуются $[8,9]$.

Так как в алгоритмах управления не рассматривается возможность возникновения устойчивых хаотических колебаний, вопрос взаимодействия активного потребителя и внешней электросистемы представляется проработанным недостаточно [10-14].

Хаотические колебания в системе электроснабжения представляют собой непредсказуемые изменения значений мощности, частоты и напряжения и являются аварийным режимом работы [15]. Одной из основных причин возникновения хаотического режима работы является наличие в электросистеме нелинейных элементов, которые при появлении сильного возму- 
щения способствуют возникновению хаотических колебаний $[16,17]$

В районах добычи нефти и газа в связи с интенсивным развитием распределенной энергетики можно прогнозировать появление активных потребителей, использующих работающие на попутном нефтяном газе газотурбинные установки. Данный тип потребителей для поставки избытка электроэнергии во внешнюю сеть может подключаться к системе электроснабжения нефтегазодобывающего предприятия. При подключении газотурбинной установки на параллельную работу с сетью возможно возникновение сильного возмущения (значительный уравнительный ток, биения и др.), которое, учитывая наличие нелинейных элементов в электросистемах активного потребителя и нефтегазодобывающего предприятия (частотно регулируемый электропривод насосов, вентильные преобразователи систем возбуждения синхронных генераторов, вторичные источники питания электроприборов жилых зданий и др.), может привести к аварийному режиму работы электросистемы устойчивым хаотическим колебаниям.

Таким образом, существует необходимость изучения вопроса подключения активного потребителя к системе электроснабжения нефтегазодобывающего предприятия. Поэтому целью работы является рассмотрение переходных процессов, возникающих при включении электрических систем активного потреби- теля и нефтегазодобывающего предприятия на параллельную работу.

Цель достигается разработкой компьютерной модели электрических систем нефтегазодобывающего предприятия и активного потребителя, моделированием переходных режимов, возникающих при включении электросистем на параллельную работу.

\section{Компьютерная модель системы электроснабжения основного электрооборудования несттегазодобывающего предприятия и активного потребителя}

Моделирование электрических систем нефтегазодобывающего предприятия и активного потребителя выполнено в специализированном программном пакете Simscape Power Systems (MatLab) [18]. Для моделирования нефтегазодобывающего предприятия выбрана система электроснабжения электротехнического комплекса основного оборудования отходящей линии для предварительной подготовки нефти (рис. 1)

Система электроснабжения является радиальной с центром питания Т1 (промысловая подстанция).

Структурная схема систем электроснабжения нефтегазодобывающего предприятия и активного потребителя в виде s-модели программного пакета MatLab показана на рис. 2.

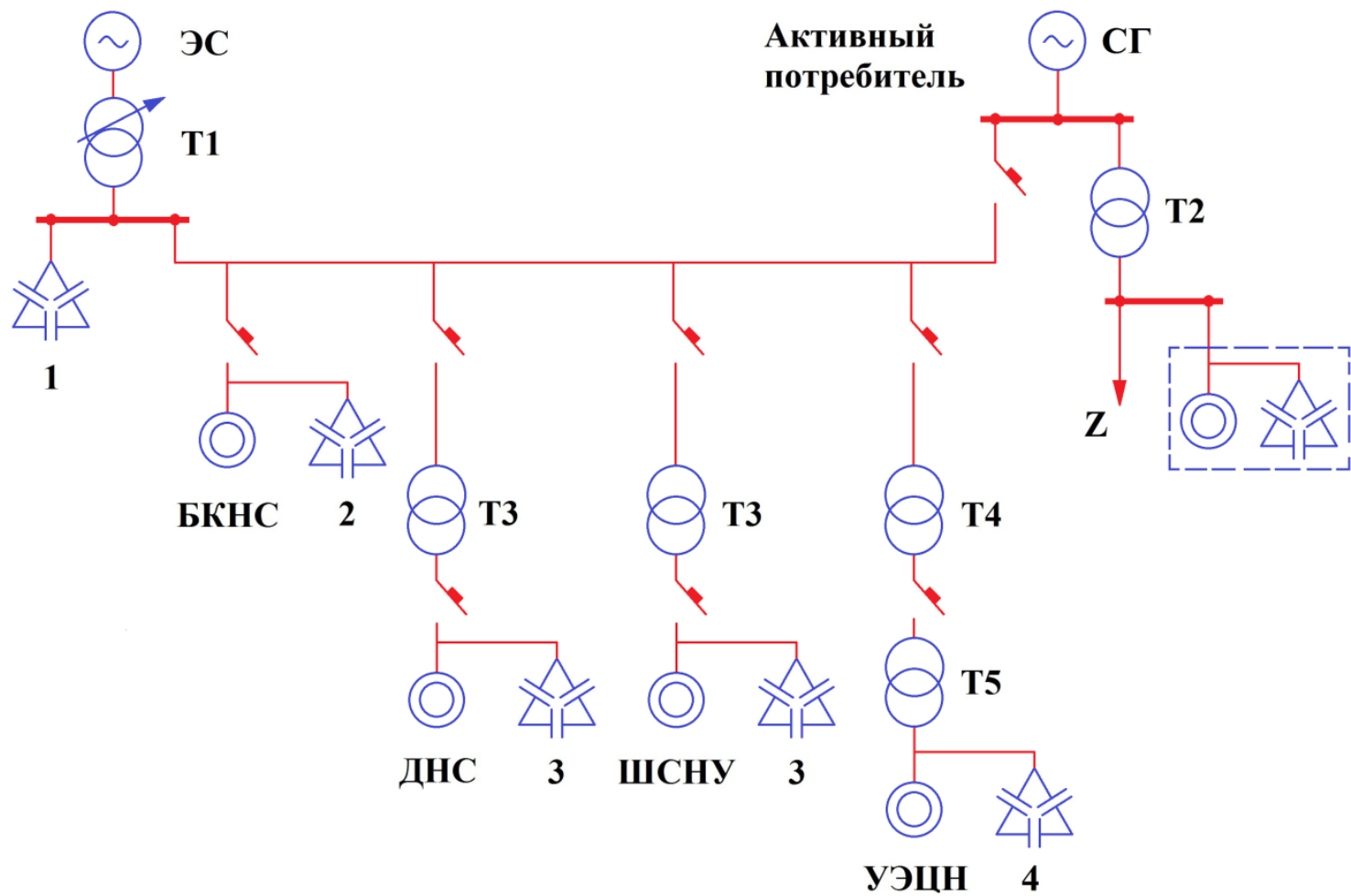

Рис. 1. Принципиальная схема системы электроснабжения нефтегазодобывающего предприятия: ЭС - внешняя электросистема; T1 - промысловая подстанция 35/6; СГ- синхронный генератор; T2, Т3, Т4 - силовые трансформаторы 6/0,4; Т5 - силовой трансформатор 0,4/2; 1-4-установки поперечной компенсации

Fig. 1. Basic circuit diagram of the power supply system of an oil and gas production enterprise: ЭC - outside electrical system; T1 - field substation 35/6; C $\Gamma$ - synchronous generator; T2, T3, T4 - power transformers 6/0,4; T5 - power transformer $0,4 / 2 ; 1-4$ - condenser banks 


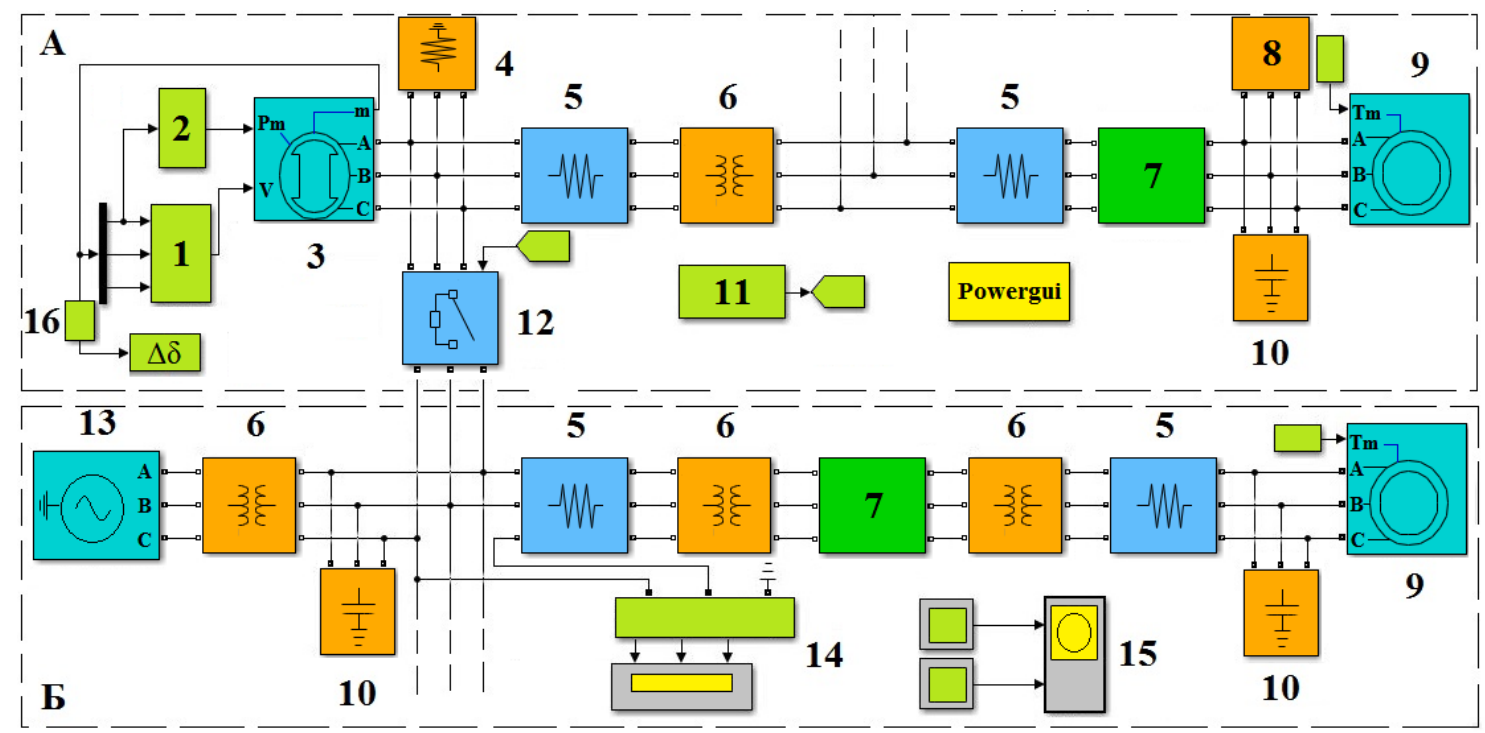

Рис. 2. Структурная схема s-модели: А - электросистема активного потребителя; Б - электросистема нефтегазодобывающего предприятия; 1 - автоматический регулятор возбуждения (тиристорная система возбуждения); 2 - автоматический регулятор частоты вращения; 3 - синхронный генератор; 4 - активная нагрузка; 5 - линия электропередачи; 6 - силовой трансформатор; 7 - преобразователь частоты; 8 - активнореактивная нагрузка; 9 - асинхронный двигатель; 10 - компенсирующее устройство; 11 - блок точной синхронизации; 12 - управляемый выключатель; 13 - внешняя электросистема; 14 - осциллограф; 15 - двухкоординатный графопостроитель; 16 - блок измерения переменных состояния электрической машины

Fig. 2. Block scheme of s-model: A - electrical system of a prosumer; 5 -electrical system of an oil and gas production enterprise; 1 -automatic excitation regulator (thyristor excitation system); 2 - turbine main governor; 3 - synchronous generator; 4 - resistive load; 5 -transmission line; 6 - power transformer; 7 -frequency converter; 8 -active-capacitive load; 9 - asynchronous machine; 10 - condenser bank; 11 - block of precision synchronization; 12 - controlled breaker; 13 - outside system; 14 - scope; 15 -XY coordinate plotter; 16 - block of machine demux measurement

Рассмотрим основные элементы электросистем нефтегазоперерабатывающего предприятия и активного потребителя в виде стандартных блоков MatLab с указанием значений параметров.

Внешняя электросистема ЭС введена в модель при помощи блока «Three-Phase Source» и имеет следующие параметры: линейное напряжение $V_{r m s}=35 \cdot 10^{3} \mathrm{~B}$, начальный сдвиг фазы А $0^{\circ}$, частота $f_{n}=50$ Гц, режим нейтрали $\mathrm{Y}_{\mathrm{g}}$, мощность трехфазного короткого замыкания $10^{13} \mathrm{BA}$, базисное напряжение $V_{\mathrm{Ph}-\mathrm{Ph}}=35 \cdot 10^{3} \mathrm{~B}$, отношение реактивного и активного сопротивлений $\mathrm{X} / \mathrm{R}=7$ [19].

Нагрузка отходящей линии представлена асинхронными двигателями (АД) блочной кустовой и дожимной насосных станций (БКНС и ДНС), штанговой скважинной насосной установки (ШСНУ) и установки электроприводного центробежного насоса (УЭЦН). Параметры АД, используемые при моделировании, рассчитаны согласно $[20,21]$ в программном пакете MathCAD (табл. 1).

С целью увеличения коэффициента мощности АД в модели используются индивидуальные и централизованные установки поперечной емкостной компенсации (УПЕК) 1-3. Расчет величины реактивной мощности УПЕК выполнен исходя из значений действующих $(0,79-0,87)$ и заданных коэффициентов мощности $(0,9-0,95)$ согласно [22]. Так как в среднем длина кабеля погружного электродвигателя составляет 2-3 км и в отходящей линии используется радиальная схема питающей сети, для увеличения уровня напряжения на клеммах центробежного насоса применена установка внутрискважинной компенсации реактивной мощности УПЕК 4 [23]. Мощности УПЕК модели отходящей линии приведены в табл. 2.

Большинство асинхронных двигателей отходящей линии подключено к шинам промысловой подстанции Т1 через понижающие трансформаторы Т3 6/0,4 и T4 6/0,4. Для управления погружным двигателем УЭЦН используется низковольтный частотный преобразователь (НПЧ), представленный в модели блоками Universal Bridge и PWM Generator. Поэтому наземное электрооборудование УЭЦН включает понижающий трансформатор Т4 6/0,4 и повышающий трансформатор Т5 0,4/2. Используемые при моделировании параметры силовых трансформаторов рассчитаны согласно [24, 25] в программном пакете MathCAD (табл. 3).

Питающая и распределительные сети отходящей линии выполнены воздушными и кабельными линиями электропередач (ЛЭП). Так как уровень напряжения не превышает 6 кВ, кабельные линии в модели представлены в виде активного сопротивления, воздушные - в виде активно-индуктивного сопротивления [26]. Марка, длина используемых при расчете проводов и кабелей, параметры схем замещения при моделировании приведены в табл. 4.

Электросистема активного потребителя состоит из неявнополюсного синхронного генератора СГ (газотурбинная электростанции ГТЭС-2,5), понижающего трансформатора Т2 и нагрузки (рис. 1). 
Таблица 1. Значения параметров асинхронного двигателя для блока Asynchronous Machine SI Units

Table 1. Asynchronous motor parameter values of the block Asynchronous Machine SI Units

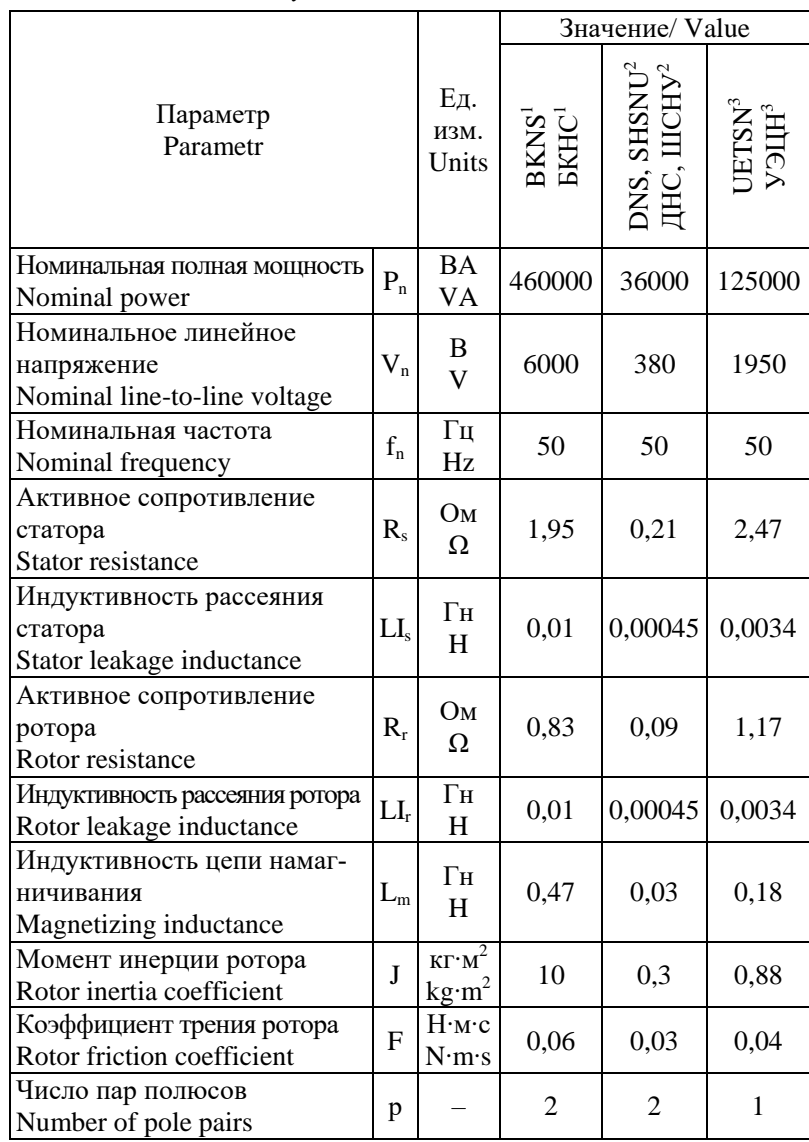

Здесь: 1 - ВАО4-450; 2 - ВАО2-72-2; 3 - ПЭД90-123.

Where: 1 - VAO4-450; 2 - VAO2-72-2; 3 - PED90-123.

Таблица 2. Значения параметров конденсаторной установки для блока Three-Phase Series RLC Load

Table 2. Condenser bank parameter values of the block Three-Phase Series RLC Load

\begin{tabular}{|c|c|c|c|c|}
\hline $\begin{array}{c}\text { Порядковый номер } \\
\text { УПЕК } \\
\begin{array}{c}\text { Serial number } \\
\text { of UPEK }\end{array}\end{array}$ & $\begin{array}{c}\text { Подстан- } \\
\text { ция } \\
\text { Substation }\end{array}$ & $\begin{array}{c}\text { БКНС } \\
\text { BKNS }\end{array}$ & $\begin{array}{c}\text { ДНС, } \\
\text { ШCHУ } \\
\text { DNS, } \\
\text { SHSNU }\end{array}$ & $\begin{array}{c}\text { УЭЦН } \\
\text { UETSN }\end{array}$ \\
\hline $\begin{array}{l}\text { Koppeкция cos } \varphi \\
\text { Power-factor correction }\end{array}$ & $0,87 \rightarrow 0,95$ & $0,86 \rightarrow 0,9$ & $0,79 \rightarrow 0,9$ & $0,84 \rightarrow 0,9$ \\
\hline $\begin{array}{l}\text { Mощность KУ, кBAp } \\
\text { Power of KU, kVAR }\end{array}$ & 150 & 50 & 10 & 15 \\
\hline
\end{tabular}

Таблица 3. Значения параметров трансформатора для блока Three-Phase Transformer

Table 3. Transformer parameter values of the block Three-Phase Transformer

\begin{tabular}{|c|c|c|c|c|c|c|}
\hline \multirow{2}{*}{\multicolumn{2}{|c|}{\begin{tabular}{|c|} 
\\
\\
Параметр \\
Parametr
\end{tabular}}} & \multirow[b]{2}{*}{$\begin{array}{l}\text { Ед. } \\
\text { изм. } \\
\text { Units }\end{array}$} & \multicolumn{4}{|c|}{$\begin{array}{l}\text { Значение } \\
\text { Value }\end{array}$} \\
\hline & & & 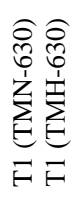 & 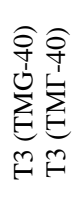 & 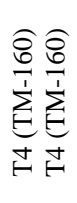 & 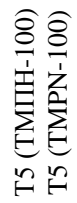 \\
\hline $\begin{array}{l}\text { Номинальная полная } \\
\text { мощность } \\
\text { Nominal power }\end{array}$ & $P_{n}$ & $\begin{array}{l}\text { кВA } \\
\mathrm{kVA}\end{array}$ & 630 & 40 & 160 & 100 \\
\hline $\begin{array}{l}\text { Номинальная частота } \\
\text { Nominal frequency }\end{array}$ & $f_{n}$ & $\begin{array}{l}\Gamma ц \\
\mathrm{~Hz}\end{array}$ & 50 & 50 & 50 & 50 \\
\hline $\begin{array}{l}\text { Напряжение первич- } \\
\text { ной обмотки } \\
\text { Winding } 1 \text { voltage }\end{array}$ & $\mathrm{V}_{1}$ & $\begin{array}{l}\kappa \mathrm{B} \\
\mathrm{kV}\end{array}$ & 35 & 6 & 6 & 0,4 \\
\hline $\begin{array}{l}\text { Напряжение вторич- } \\
\text { ной обмотки } \\
\text { Winding } 2 \text { voltage }\end{array}$ & $\mathrm{V}_{2}$ & кВ & 6 & 0,4 & 0,4 & 2 \\
\hline $\begin{array}{l}\text { Активное сопротивле- } \\
\text { ние первичной и вто- } \\
\text { ричной обмоток } \\
\text { Winding } 1,2 \text { resistance }\end{array}$ & $\mathrm{R}_{1}, \mathrm{R}_{2}$ & $\begin{array}{c}\text { o.e. } \\
\text { pu }\end{array}$ & 0,006 & 0,012 & 0,008 & 0,01 \\
\hline $\begin{array}{l}\text { Индуктивное сопро- } \\
\text { тивление первичной и } \\
\text { вторичной обмоток } \\
\text { Winding } 1,2 \text { inductance }\end{array}$ & $\mathrm{L}_{1}, \mathrm{~L}_{2}$ & $\begin{array}{c}\text { o.e. } \\
\text { pu }\end{array}$ & 0,029 & 0,019 & 0,02 & 0,026 \\
\hline $\begin{array}{l}\text { Активное сопротивле- } \\
\text { ние ветви намагничи- } \\
\text { вания } \\
\text { Magnetizing resistance }\end{array}$ & $\mathrm{R}_{\mathrm{m}}$ & $\begin{array}{c}\text { o.e. } \\
\text { pu }\end{array}$ & 11,27 & 2,6 & 7,79 & 14,79 \\
\hline $\begin{array}{l}\text { Индуктивное сопро- } \\
\text { тивление ветви намаг- } \\
\text { ничивания } \\
\text { Magnetizing inductance }\end{array}$ & $\mathrm{L}_{\mathrm{m}}$ & $\begin{array}{c}\text { o.e. } \\
\text { pu }\end{array}$ & 76,09 & 19,83 & 52,05 & 69,88 \\
\hline
\end{tabular}

Неявнополюсный синхронный генератор представлен в модели блоком Simplified Synchronous Machine SI Units [27]: номинальная полная мощность $P_{n}=2,5 \cdot 10^{6} \mathrm{BA}$, номинальное напряжение $U_{n}=6300 \mathrm{~B}$, номинальная частота $f_{n}=50$ Гц, маховый момент ротоpa $J=680 \kappa{ }^{*} \mathrm{~m}^{2}$, число пар полюсов $\mathrm{p}=1$, активное сопротивление и индуктивность обмотки статора соответственно $R=0,04$ Ом и $L=0,022$ Гн. При помощи блока Three-Phase Series RLC Load имитируется отбор мощности на собственные нужды генератора: активная мощность $P_{n}=40$ кВт, номинальное напряжение $U_{n}=6300 \mathrm{~B}$, частота $f_{n}=50$ Гц.

Таблица 4. Значения параметров линии электропередач для блока Three-Phase Series RLC Branch

Table 4. Transmission line parameter values of the block Three-Phase Series RLC Branch

\begin{tabular}{|c|c|c|c|c|c|}
\hline $\begin{array}{c}\text { Активный потребитель } \\
\text { Prosumer }\end{array}$ & $\begin{array}{l}\text { БKHC } \\
\text { BKNS }\end{array}$ & \multicolumn{2}{|c|}{$\begin{array}{l}\text { ДHC, ШCHУ } \\
\text { DNS, SHSNU }\end{array}$} & \multicolumn{2}{|c|}{$\begin{array}{l}\text { УЭЦН } \\
\text { UETSN }\end{array}$} \\
\hline ВЛ 6 кВ & ВЛ 6 кВ & ВЛ 6 кВ & ВЛ 0,4 кВ & ВЛ 6 кВ & КЛ 2 кВ \\
\hline VL $6 \mathrm{kV}$ & VL $6 \mathrm{kV}$ & VL $6 \mathrm{kV}$ & VL $0.4 \mathrm{kV}$ & VL $6 \mathrm{kV}$ & KL $2 \mathrm{kV}$ \\
\hline СИП-3 1×35 & СИП-3 1×16 & СИП-3 $1 \times 16$ & СИП-2 3×16 & СИП-3 $1 \times 16$ & КПБП $3 \times 16$ \\
\hline SIP-3 $1 \times 35$ & SIP-3 $1 \times 16$ & SIP-3 $1 \times 16$ & SIP-2 $3 \times 16$ & SIP-3 $1 \times 16$ & KPBP $3 \times 16$ \\
\hline $\mathrm{r}_{0}=0,868$ & $\mathrm{r}_{0}=1,91$ & $\mathrm{r}_{0}=1,91$ & $\mathrm{r}_{0}=1,91$ & $\mathrm{r}_{0}=1,91$ & $\mathrm{r}_{0}=1,15$ \\
\hline $\mathrm{x}_{0}=0,37$ & $\mathrm{x}_{0}=0,39$ & $x_{0}=0,39$ & $\mathrm{x}_{0}=0,39$ & $\mathrm{x}_{0}=0,39$ & $\mathrm{x}_{0}=0,41$ \\
\hline $\mathrm{L}=1$ & $\mathrm{~L}=0,5$ & $\mathrm{~L}=1$ & $\mathrm{~L}=1,5$ & $\mathrm{~L}=2$ & $\mathrm{~L}=3$ \\
\hline
\end{tabular}

Здесь: ВЛ - воздушная ЛЭП, КЛ - кабельная ЛЭП, $r_{0}$ и $x_{0}$ - удельное активное и реактивное сопротивления ЛЭП

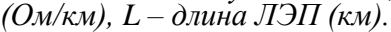

Where: VL - overhead $L E P, K L$ - underground $L E P, r_{0}$ and $x_{0}-L E P$ resistance and reactance per unit length $(\Omega / \mathrm{km}), L-$ length of $L E P(\mathrm{~km})$. 
Структурные схемы автоматических регуляторов напряжения и частоты вращения синхронного генератора (АРВ и АРЧВ), значения коэффициентов усиления и постоянных времени выбраны согласно данным работы [28]. АРЧВ выполнен на основе пропорционально-интегрально-дифференциального звена. АРВ представлен тиристорной системой возбуждения. Для оценки значений механической мощности турбины $P_{m}$ и напряжения на обмотке возбуждения $V_{f}(0,75$ o.e. и 1,31 о.е. соответственно) использована функция блока Powergui Machine Initialisation.

Понижающий трансформатор Т2 представлен в модели блоком Three-Phase Transformer со следующими параметрами: $P_{n}=1000$ кВА, $f_{n}=50$ Гц, $V_{1}=6$ кВ, $V_{2}=0,4 \kappa \mathrm{B}, R_{1}=R_{2}=0,006$ o.e., $L_{1}=L_{2}=0,022$ o.e., $R_{m}=200$ o.e., $L_{m}=267$ o.e.

Двигательная нагрузка составляет 60 \% от общей нагрузки активного потребителя и представлена в модели асинхронными двигателями ВАО2-72-2 (табл. 1) суммарной мощностью 600 кВт. Для повышения коэффициента мощности двигателей использована индивидуальная компенсацией реактивной мощности (табл. 2). Статическая часть нагрузки задана комплексным сопротивлением.

\section{Моделирование подключения активного потребителя к системе электроснабжения нефтегазодобывающего предприятия}

При моделировании подключения активного потребителя к системе электроснабжения отходящей линии используется метод точной синхронизации. Метод точной синхронизации подразумевает соответствие в момент включения частот, амплитуды и фаз напряжений генератора и внешней сети [29]. При моделировании в MatLab точная синхронизация реализуется управляемым трехфазным выключателем Three-Phase Circuit Breaker. Условия точной синхронизации выполняются в модели при помощи логических операторов Relational Operator, позволяющих сравнивать значения амплитуды, частоты и фазы напряжений генератора и электросистемы отходящей линии. Для соответствия моделируемого процесса синхронизации существующим автоматическим системам включения турбогенераторов на параллельную работу с сетью логические операторы модели допускают следующие погрешности [30]: различие амплитуд напряжений сети и генератора не более $1 \%$; различие фаз напряжений сети и генератора не более $10 \%$; различие частот сети и генератора не более $0,1 \%$.

Как показано на рис. 3, при включении газотурбинной установки активного потребителя на параллельную работу с использованием метода точной синхронизации возникает незначительное возмущение длительностью 4 с в пределах флуктуаций параметров электросистемы при нормальном переходном режиме [31]. Возникновение переходного процесса вызвано введенными в компьютерную модель погрешностями условий синхронизации, нарушением баланса генерируемой и потребляемой мощностей.

Аварийный режим, возникающий при несинхронном подключении генератора активного потребителя к электросистеме отходящей линии, демонстрирует устойчивые хаотические колебания [32] (рис. 4).

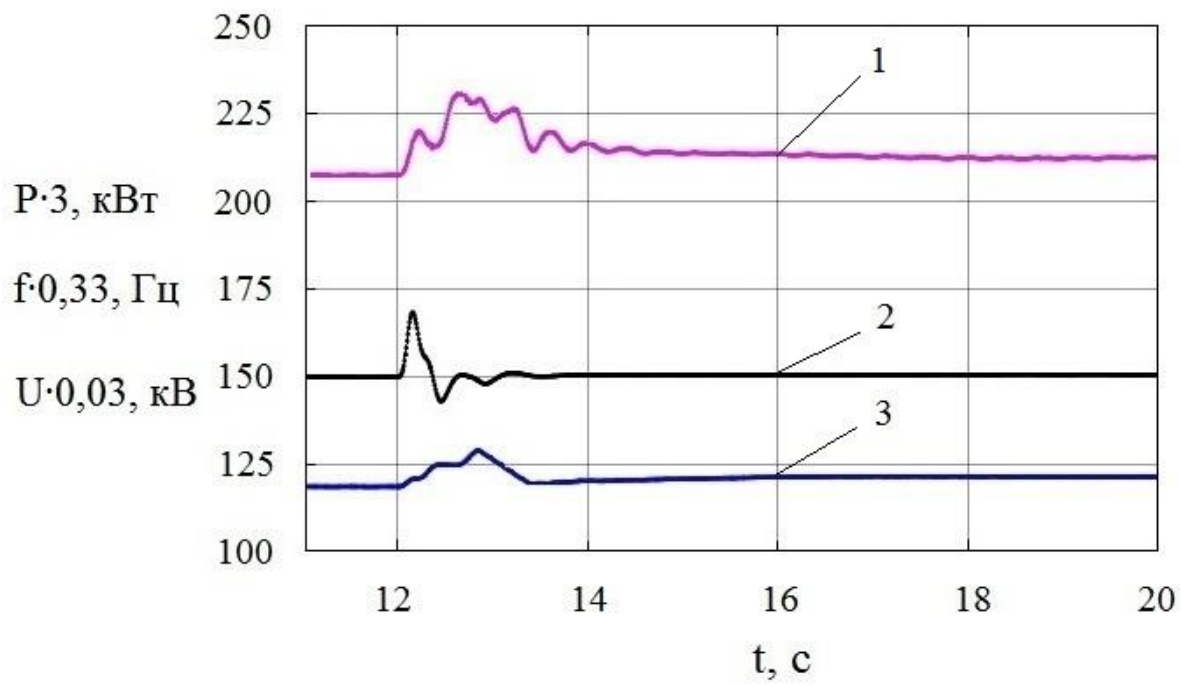

Рис. 3. Нормальный переходный режим в электросистеме (промысловая подстанция T1 со стороны низкого напряжения): 1 - активная мощность, 2 - частота, 3 - фазное напряжение

Fig. 3. Normal transitional mode in the electrical system (field substation T1 from low voltage level): 1 - active power; 2 frequency; 3 - line to ground voltage 


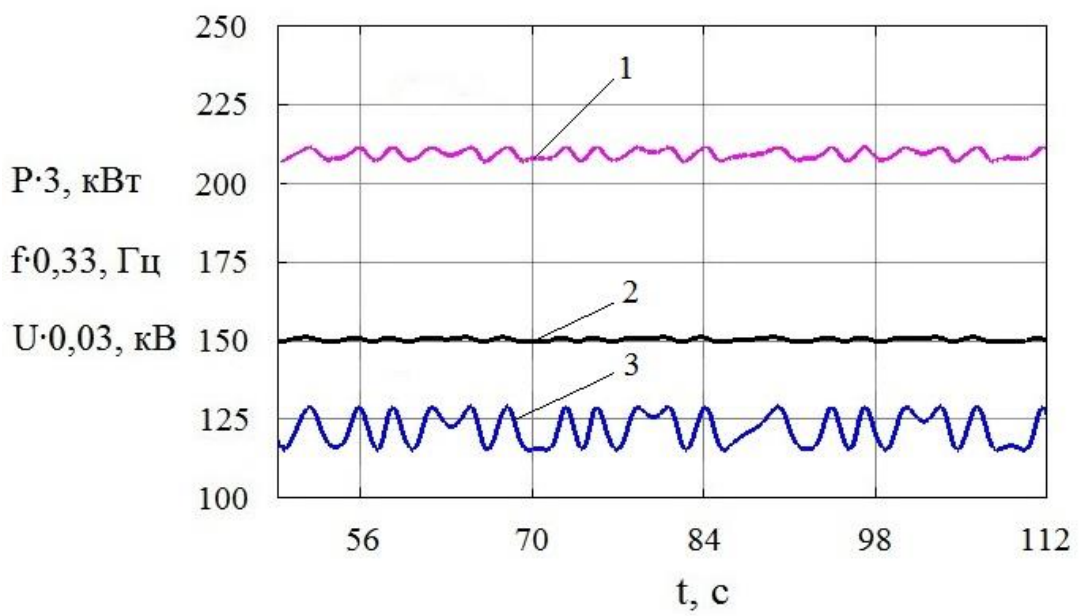

Pис. 4. Переходный режим с возникновением устойчивых хаотических колебаний (промысловая подстанция T1 со стороны низкого напряжения): 1 - активная мощность, 2 - частота, 3 - фазное напряжение

Fig. 4. Transitional mode in the electrical system of outgoing line (field substation T1 from low voltage level): 1 - active power; 2 -frequency; 3 - line to ground voltage

Как видно из рисунка, при хаотическом режиме работы на шинах промысловой подстанции диапазон изменений значений активной мощности составляет 18 кВт, частоты - 1,5 Гц, напряжения - 400 В.

Согласно [33] в точке общего присоединения к электрической сети нормально допустимое значение установившегося отклонения напряжения $\delta U_{\text {y }}$ составляет $\pm 5 \%$, предельно допустимое отклонение частоты $\Delta f$ составляет $\pm 0,4$ Гц, к пульсациям мощности требования не предъявляются. Исходя из результатов компьютерного моделирования $\delta U_{\mathrm{y}}= \pm 6,7 \%, \Delta f= \pm 1,5$ Гц. Таким образом, при хаотических колебаниях на шинах промысловой подстанции установившееся отклонение напряжения превышает нормально допустимое значение на $1,7 \%$, а отклонение частоты превышает предельно допустимое значение на 1,1 Гц.

Следует отметить требования к качеству электроэнергии питающей сети со стороны основного электроприемника нефтегазодобывающего предприятия асинхронного двигателя. Согласно [34] нормирую- щий уровень помехоустойчивости асинхронного дви-

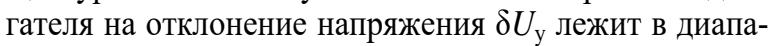
зоне от $-5 \%$ до $+10 \%$. Результаты моделирования показывают возможность выхода $\delta U_{\text {y }}$ за нижнюю границу диапазона. Пониженный уровень напряжения питания асинхронного двигателя вызывает увеличение потерь в «меди» и способствует его перегреву. Также снижение напряжения питания может привести к потере двигателем устойчивости и «опрокидыванию». Пульсациям сетевого напряжения и частоты соответствуют колебания вращающего момента асинхронного двигателя. Нестабильность вращающего момента может явиться причиной застопоривания механических систем оборудования нефтегазодобывающего предприятия и способствует их повышенному износу.

Фазовый портрет изменения напряжения на шинах промысловой подстанции Т1 (рис. 5) соответствует нестранному хаотическому аттрактору.

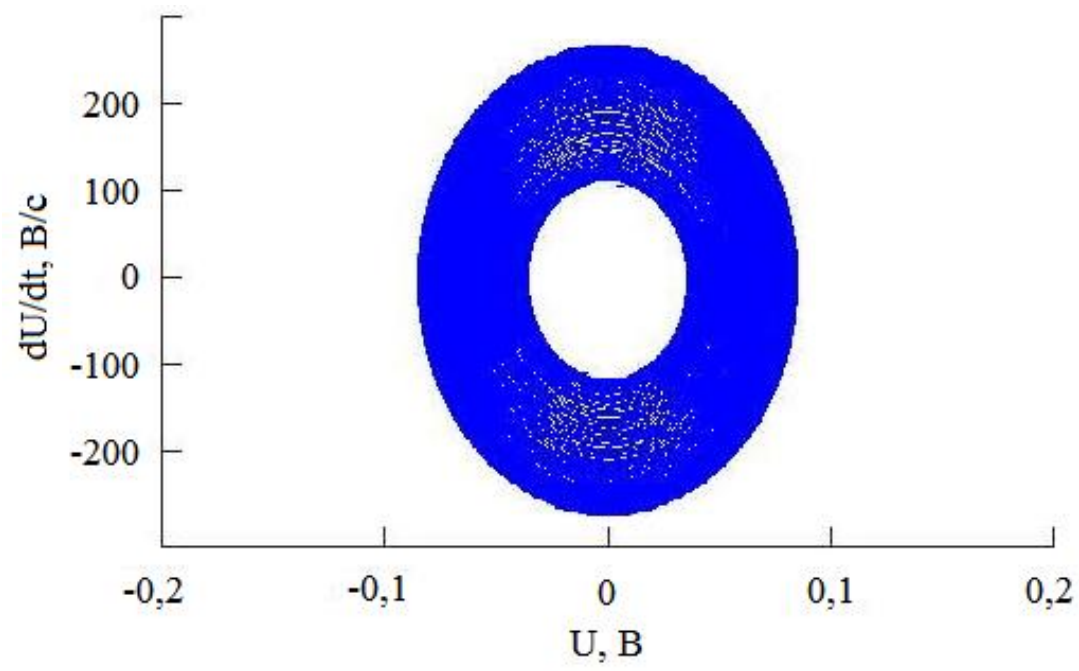

Pис. 5. Фазовый портрет напряжения на шинах промысловой подстанции $T 1$ со стороны низкого напряжения Fig. 5. Phase portrait of the field substation T1 bus voltage from low voltage level 
Для аварийного режима работы выполнен расчет старшего показателя Ляпунова $\lambda$ по следующей формуле [35, 36]:

$$
\lambda=\frac{1}{N \Delta T} \cdot \sum_{k=1}^{N} \ln \left|\Delta \bar{\delta}^{k \Delta T}\right|,
$$

где $N$ - число точек измерения; $\Delta T$ - шаг дискретизации; $\Delta \delta$ - отклонение угла ротора генератора активного потребителя.

В существующих электросистемах для реализации расчета старшего показателя Ляпунова целесообразно использовать систему синхронизированных векторных измерений WAMS. Данная система позволяет измерять величину угла на генераторах в режиме реального времени. Типичным режимом функционирования WAMS является импульсный режим: интервал измерения $1 \mathrm{c}$, период квантования 1 мс. В этой связи приняты следующие значения параметров для расчета показателя Ляпунова: $\Delta \mathrm{T}=1 \mathrm{mc}, \mathrm{N}=10^{3}$. В модели отклонение угла ротора генератора $\Delta \delta$ вычисляется при помощи блока Machines Measurement Demux (рис. 2). Блок to Workspace с форматом сохранения данных Time Series (рис. 2) формирует матрицу значений $\Delta \delta$. На основе матрицы значений $\Delta \delta(\Delta \delta$.data и $\Delta \delta$.time $)$ в командном окне MatLab по указанной выше формуле вычисляется старший показатель Ляпунова. В результате расчета получено значение старшего показателя Ляпунова 0,44. Наличие положительного показателя Ляпунова подтверждает хаотический характер колебаний напряжения, мощности и частоты в электросистеме.

Результаты моделирования коррелируют с исследованиями [37], согласно которым на шинах трансформаторной подстанции в течение не менее 8 минут наблюдались хаотические колебания напряжения. Исходя из осциллограмм фазных напряжений, откло-

\section{СПИСОК ЛИТЕРАТУРЬ}

1. Лукутин Б.В., Муравлев И.О., Плотников И.А. Системы электроснабжения с ветровыми и солнечными электростанциями. - Томск: Томский политехнический университет, 2015. $120 \mathrm{c}$.

2. Sawle Y., Gupta S.C., Bohre A.K. Review of hybrid renewable energy systems with comparative analysis of off-grid hybrid system // Renewable and Sustainable Energy Reviews. - 2018. V. 81. - Iss. 2. - P. 2217-2235.

3. Micro cogeneration: towards decentralized energy systems M. Pehnt, M. Cames, C. Fischer, B. Praetorius, L. Schneider, K. Schumacher, J.-P. Vob. - Heidelberg: Springer-Verlag, 2006. $346 \mathrm{p}$.

4. A hybrid renewable system based on wind and solar energy coupled with an electrical storage: dynamic simulation and economic assessment / A. Buonomano, F. Calise, M.D. d'Accadia, M. Vicidomini // Energy. - 2018. - V. 155. - P. 174-189.

5. Двухконтурный накопитель энергии для гибридных энергетических систем с возобновляемыми источниками энергии / С.Г. Обухов, И.А. Плотников, Ибрагим Ахмед, В.Г. Масолов // Известия Томского политехнического университета. Инжиниринг георесурсов. - 2020. - Т. 331. - № 1. - С. 64-76.

6. Балабанов М.С., Бабошкина С.В., Хамитов Р.Н. Экологические аспекты в энергосберегающей политике на этапе создания в России интеллектуальных энергосистем с активноадаптивной сетью // Известия Томского политехнического универ- нение от номинального значения частоты составляет 1--2 Гц, амплитуды - 50-59\%.

\section{Заключение}

Рассмотрено включение активного потребителя на параллельную работу с системой электроснабжения нефтегазодобывающего предприятия.

Для моделирования переходных процессов разработана компьютерная модель электросистем основного оборудования отходящей линии для предварительной подготовки нефти и активного потребителя.

Использование метода точной синхронизации при подключении генератора активного потребителя к электросистеме отходящей линии вызывает нормальный переходный режим с длительностью возмущения $4 \mathrm{c}$.

При несинхронном включении генератора активного потребителя на параллельную работу с системой электроснабжения отходящей линии характер изменения параметров электросистемы демонстрирует устойчивые хаотические колебания. При данном режиме работы на шинах промысловой подстанции диапазон изменений значений активной мощности составляет 18 кВт, частоты - 1,5 Гц, напряжения 400 В. Фазовый портрет напряжения на шинах промысловой подстанции является нестранным аттрактором с положительным старшим показателем Ляпунова 0,44 , что подтверждает возникновение хаотического режима работы.

Условием возникновения хаотического режима работы является присутствие в электросистеме нелинейных элементов, таких как газотурбинная установка активного потребителя и мощные преобразователи частоты электропривода насосов, а импульсом для развития хаоса служит несинхронное включение генератора активного потребителя.

ситета. Инжиниринг георесурсов. - 2015. - Т. 326. - № 11. C. 141-152.

7. Kakran S., Chanana S. Smart operations of smart grids integrated with distributed generation: a review // Renewable and Sustainable Energy Reviews. - 2018. - V. 81 (1). - P. 524-535.

8. Towards the next generation of smart grids: semantic and holonic multi-agent management of distributed energy resources / S. Howell, Y. Rezgui, J.-L. Hippolyte, B. Jayan, H. Li // Renewable and Sustainable Energy Reviews. - 2017. - V. 77. P. 193-214.

9. Dehkordi N.M., Sadati N., Hamzeh M. Fully distributed cooperative secondary frequency and voltage control of islanded microgrids // IEEE Transactions on Energy Conversion. - 2017. V. 32. - Iss. 2. - P. 675-685.

10. Raju L., Milton R.S., Mahadevan S. Multi agent systems based distributed control and automation of micro-grid using MACSimJX // 10th International Conference on Intelligent Systems and Control (ISCO). - Coimbatore, 2016. - P. 1-6.

11. Фишов А.Г., Мукатов Б.Б. Реконфигурация электрических сетей с распределенной генерацией и мультиагентным управлением // Известия Томского политехнического университета. Инжиниринг георесурсов. - 2015. - Т. 326. - № 9. - С. 143-152.

12. Marah R., Hibaoui A.E. Algorithms for Smart Grid management // Sustainable Cities and Society. - 2018. - V. 38. - P. 627-635.

13. MAS-based distributed coordinated control and optimization in microgrid and microgrid clusters: a comprehensive overview / Y. Han, K. Zhang, H. Li, E.A.A. Coelho, J.M. Guerrero // IEEE 
Transactions on Power Electronics. - 2018. - V. 33. - Iss. 8. P. 6488-6508.

14. Survey of multiagents systems application in Microgrids / F.Z. Harmouch, N. Krami, D. Benhaddou, N. Hmina, E. Zayer, E.H. Margoum // International Conference on Electrical and Information Technologies (ICEIT). - Tangier, 2016. - P. 270-275.

15. Детерминированный хаос в нелинейных электрических цепях и системах / В.К. Федоров, В.К. Грунин, П.В. Рысев, Е.Ю. Свешникова. - Омск: Омский научный вестник, 2006. $130 \mathrm{c}$.

16. Chaos in a simple power system / H.D. Chiang, C.W. Liu, P.P. Varaiya, F.F. Wu, M.G. Lauby // IEEE Transactions on Power Systems. - 1993. - V. 8. - Iss. 4. - P. 1407-1417.

17. Power system instability and chaos / Y. Yu, H. Jia, P. Li, J. Su // Electric power systems research. - 2003. - V. 65. - Iss. 3. P. $187-195$.

18. Архипова О.В., Ковалев В.З., Хамитов Р.Н. Методика моделирования регионально обособленного электротехнического комплекса // Известия Томского политехнического университета. Инжиниринг георесурсов. - 2019. - Т. 330. - № 1. C. $173-180$.

19. Зарудная А.П., Горшков К.Е. Особенности применения пакета MATLAB/Simulink для анализа статической устойчивости синхронных генераторов в энергосистеме // Вестник ЮжноУральского государственного университета. Серия: Энергетика. - 2017. - Т. 17. - № 3. - С. 43-54.

20. Боловин Е.В., Глазырин А.С. Метод идентификации параметров погружных асинхронных электродвигателей установок электроприводных центробежных насосов для добычи нефти // Известия Томского политехнического университета. Инжиниринг георесурсов. - 2017. - Т. 328. - № 1. - С. 123-131.

21. Герман-Галкин С.Г., Кардонов Г.А. Электрические машины: Лабораторные работы на ПК. - СПб.: Корона принт, 2003. $256 \mathrm{c}$.

22. Марикин А.Н., Мирощенко А.В., Кузьмин С.В. Устройство поперечной компенсации реактивной мощности с изменяющейся индуктивностью // Известия Петербургского университета путей сообщения. - 2015. - Т. 44. - № 3. - С. 77-84.

23. Влияние внутрискважинного компенсатора на падение напряжения в элементах электротехнического комплекса добывающей скважины / В.А. Копырин, О.В. Смирнов, А.Л. Портнягин, Р.Н. Хамитов // Известия Томского политехнического университета. Инжиниринг георесурсов. - 2018. T.329. - № 9. - С. 117-124.

24. Новаш И.В., Румянцев Ю.В. Расчет параметров модели трехфазного трансформатора из библиотеки MatLab-Simulink c учетом насыщения магнитопровода // Энергетика. Известия высших учебных заведений и энергетических объединений СНГ. - 2015. - № 9. - С. 12-24.
25. Косарев Б.А., Федоров В.К. Модель электротехнической системы с распределенной генерацией // Омский научный вестник. -2019. - Т. 167. - № 5. - С. 64-71.

26. Кабельные и воздушные линии электропередачи / Н.П. Бадалян, Г.П. Колесник, Д.П. Андрианов, Ю.С. Чебрякова. - Владимир: Изд-во ВлГУ, 2019. - 260 с

27. Моделирование неявнополюсного синхронного генератора в Matlab / М.М. Файзиев, Н.А. Курбанов, А.Б. Имомназаров, Б.С. Бобоназаров, А.Э. Бекишев // Вестник науки и образования. - 2017. - Т. 1. - № 5 (29). - С. 10-14.

28. Булатов Ю.Н., Крюков А.В., Хынг Ч.З. Автоматические регуляторы для установок распределенной генерации // Системы. Методы. Технологии. - 2014. - Т. 23. - № 3. - С. 108-116.

29. Веников В.А. Переходные электромеханические процессы в электрических системах. - М.: Высшая школа, 1985. - 536 с.

30. Гайсаров Р.В. Режимы работы электрооборудования электрических станций и подстанций: Часть 1. Режимы работы синхронных генераторов и компенсаторов. - Челябинск: Изд-во ЮУрГУ, 2005. - 42 c.

31. Илюшин П.В., Куликов А.Л. Автоматика управления нормальными и аварийными режимами энергорайонов с распределенной генерацией. - Н. Новгород: НИУ РАНХиГС, 2019. $364 \mathrm{c}$.

32. Vahdati P.M., Kazemi A. Bifurcations and chaos in nonlinear dynamics of power systems // 24th Iranian Conference on Electrical Engineering (ICEE). - Shiraz, 2016. - P. 1706-1711.

33. ГОСТ 32144-2013 Электрическая энергия. Совместимость технических средств электромагнитная. Нормы качества электрической энергии в системах электроснабжения общего назначения. Межгосударственный стандарт. - М.: Стандартинформ, 2014. $-20 \mathrm{c}$

34. ГОСТ Р 50034-92 Совместимость технических средств электромагнитная. Двигатели асинхронные напряжением до 1000 B. Нормы и методы испытаний на устойчивость к электромагнитным помехам. - М.: Изд-во стандартов, 2004. - 8 с.

35. Рысев П.В., Рысев Д.В., Федоров В.К., Шульга К.С., Прусс С.Ю. Идентификация и моделирование хаотических режимов в электроэнергетических системах // Динамика систем, механизмов и машин: Материалы одиннадцатой Международной IEEE научно-технической конференции. - Омск: ОмГТУ, 2017. - Т. 5. - № 3. - С. 101-107.

36. Determining Lyapunov exponents from a time series / A. Wolf, J.B. Swift, H.L. Swinney, J.A. Vastano // Physica D: Nonlinear Phenomena. - 1985. - V. 16. - Iss. 3. - P. 285-317.

37. Mork B.A. Understanding and dealing with ferroresonance // Minnesota Power Systems Conference. - St. Paul, 2006. - P. 1-10.

Поступила 05.08.2020 2.

\section{Информация об авторах}

Косарев Б.А., инженер кафедры электрической техники Энергетического института Омского государственного технического университета.

Федоров В.К., доктор технических наук, профессор, профессор кафедры электроснабжения промышленных предприятий Энергетического института Омского государственного технического университета.

Хамитов P.H., доктор технических наук, доцент, профессор кафедры электрической техники Энергетического института Омского государственного технического университета; профессор кафедры электроэнергетики Института промышленных технологий и инжиниринга Тюменского индустриального университета. 
UDC 621.311

\title{
MODELING THE CONNECTION OF A PROSUMER IN PARALLEL OPERATION WITH THE POWER SUPPLY SYSTEM OF AN OIL AND GAS PRODUCTION ENTERPRISE
}

\author{
Boris A. Kosarev1, \\ BorisK_88@mail.ru
}

Vladimir K. Fedorov ${ }^{1}$ el.tech.omgtu@gmail.com

\author{
Rustam N. Khamitov ${ }^{1,2}$, \\ apple_27@list.ru \\ 1 Omsk State Technical University, \\ 11, Mira Avenue, Omsk, 644050, Russia. \\ 2 Tyumen Industrial University, \\ 38, Volodarsky street, Tyumen, 625000, Russia
}

Relevance of the research. In the areas of oil and gas production in relation to the intensive development of distributed generation, it is possible to predict the emergence of prosumers using gas turbine units running on associated petroleum gas. At the same time, the question of transient processes arising when a prosumer is connected to the power supply system of an oil and gas production enterprise seems to be little studied. As a result of the non-synchronous activation of the gas turbine installation of a prosumer, transients can turn into steady chaotic oscillations - electrical system emergency state. Thus, there is a need to study the connection of a prosumer to centralized power supply systems in oil and gas production areas.

The aim of the research is to investigate the transient processes occurring when connecting a prosumer electrical system in parallel operation with oil and gas production enterprise electrical system.

Objects: electrical systems of the oil and gas production enterprise and the prosumer.

Methods. Transient processes in electrical power systems of a prosumer and an oil and gas production enterprise are investigated by computer simulation in the software package Simscape Power Systems (Matlab). Theoretical foundations of power supply for industrial enterprises were used in developing the circuit diagrams of electrical systems and determining the power of elements and voltage levels. Theoretical foundations of electrical engineering and electrical-machine theory were used in determining the system component parameter values.

Results. The simulation results show a normal transitional mode lasting $4 s$ when the prosumer generator is switched on by the precision synchronization method for parallel operation with an outside system. When the generator is switched on asynchronously, the electrical system shows steady chaotic oscillations. Chaotic oscillations are indicated by the aperiodic nature of the change in time of the values of frequency, voltage, and power, as well as the non-strange chaotic attractor with a positive Lyapunov exponent of 0,44. Chaotic operating regime shows the field substation bus power range $18 \mathrm{~kW}$, frequency range $1,5 \mathrm{~Hz}$, voltage range $400 \mathrm{~V}$.

\section{Key words:}

Dispersed generation, prosumer, chaotic oscillations, strange non-chaotic attractor, Lyapunov exponent, computer simulation.

\section{REFERENCES}

1. Lukutin B.V., Muravlev I.O., Plotnikov I.A. Sistemy elektrosnabzheniya s vetrovymi $i$ solnechnemi electrostantsiyami [Power supply systems with wind and solar power plants]. Tomsk, Tomsk Polytechnic University Publ., 2015. 120 p.

2. Sawle Y., Gupta S.C., Bohre A.K. Review of hybrid renewable energy systems with comparative analysis of off-grid hybrid system. Renewable and Sustainable Energy Reviews, 2018, vol. 81, no. 2, pp. 2217-2235.

3. Pehnt M., Cames M., Fischer C., Praetorius B., Schneider L., Schumacher K., Vob J.-P. Micro cogeneration: towards decentralized energy systems. Heidelberg, Springer-Verlag, 2006. 346 p.

4. Buonomano A., Calise F., D'accadia M.D., Vicidomini M. A hybrid renewable system based on wind and solar energy coupled with an electrical storage: dynamic simulation and economic assessment. Energy, 2018, vol. 155, pp. 174-189.

5. Obukhov S.G., Plotnikov I.A., Ibrahim A., Masolov V.G. Dua energy storage for hybrid energy systems with renewable energy sources. Bulletin of the Tomsk Polytechnic University. Geo Asset Engineering, 2020, vol. 331, no. 1, pp. 64 - 76. In Rus.

6. Balabanov M.S., Baboshkina S.V., Khamitov R.N. Electric saving and environmental aspects in policy at the stage of smart grid creation in Russia. Bulletin of the Tomsk Polytechnic University. Geo Assets Engineering, 2015, vol. 326, no. 11, pp. 141-152. In Rus.
7. Kakran S., Chanana S. Smart operations of smart grids integrated with distributed generation: a review. Renewable and Sustainable Energy Reviews, 2018, vol. 81, pp. 524-535.

8. Howell S., Rezgui Y., Hippolyte J.-L., Jayan B., Li H. Towards the next generation of smart grids: Semantic and holonic multiagent management of distributed energy resources. Renewable and Sustainable Energy Reviews, 2017, vol. 77, pp. 193-214

9. Dehkordi N.M., Sadati N., Hamzeh M. Fully distributed cooperative secondary frequency and voltage control of islanded microgrids. IEEE Transactions on Energy Conversion, 2017, vol. 32, no. 2 , pp. $675-685$.

10. Raju L., Milton R.S., Mahadevan S. Multi agent systems based distributed control and automation of micro-grid using MACSimJX. $10^{\text {th }}$ International Conference on Intelligent Systems and Control (ISCO). Coimbatore, 2016. pp. 1-6.

11. Fishov A.G., Mukatov B.B. Reconfiguration of electric networks with distributed generation and multiagent control. Bulletin of the Tomsk Polytechnic University. Geo Assets Engineering, 2015, vol. 326, no. 9, pp. 143-152. In Rus.

12. Marah R., Hibaoui A.E. Algorithms for Smart Grid management. Sustainable Cities and Society, 2018, vol. 38, pp. 627-635.

13. Han Y., Zhang K., Li H., Coelho E.A.A., Guerrero J.M. MASbased distributed coordinated control and optimization in microgrid and microgrid clusters: a comprehensive overview. IEEE Transactions on Power Electronics, 2018, vol. 33, no. 8, pp. 6488-6508. 
14. Harmouch F.Z., Krami N., Benhaddou D., Hmina N., Zayer E., Margoum E.H. Survey of multiagents systems application in Microgrids. International Conference on Electrical and Information Technologies (ICEIT). Tangier, 2016. pp. 270-275.

15. Fedorov V.K., Grunin V.K., Rysev P.V., Sveshnikova E.Yu. Determinirovanny khaos $v$ nelineynykh elektricheskikh tsepyakh sistemakh [Deterministic chaos in nonlinear electrical circuits and systems]. Omsk, Omsk Scientific Bulletin Publ., 2006. 130 p.

16. Chiang H.D., Liu C.W., Varaiya P.P., Wu F.F., Lauby M.G. Chaos in a simple power system. IEEE Transactions on Power Systems, 1993, vol. 8, no. 4, pp. 1407-1417.

17. Yu Y., Jia H., Li P., Su J. Power system instability and chaos. Electric power systems research, 2003, vol. 65, no. 3, pp. 187-195.

18. Arkhipova O.V., Kovalev V.Z., Khamitov R.N. Methodology of modeling regionally isolated electrotechnical complex. Bulletin of the Tomsk Polytechnic University. Geo Assets Engineering, 2019, vol. 330, no. 1, pp. 173-180. In Rus.

19. Zarudnaya A.P., Gorshkov K.E. Features of package MATLAB/SIMULINK application for analyzing static sustainability of synchronous generators in power system. News South Ural State University. Power engineering series, 2017, vol. 17, no. 3 , pp. 43-54. In Rus.

20. Bolovin E.V., Glazyrin A.S. Method for identifying parameters of submersible induction motors of electrical submersible pump units for oil production. Bulletin of the Tomsk Polytechnic University. Geo Assets Engineering, 2017, vol. 328, no. 1, pp. 123-131. In Rus.

21. German-Galkin S.G., Kardonov G.A. Electricheskie mashiny: laboratornye raboty na PC [Electrical machines: laboratory work for PC]. St-Petersburg, Korona print, 2003. $256 \mathrm{p}$.

22. Marikin A.N., Miroshchenko V.A., Kuzmin S.V. Reactive power shunt compensation device with variable inductance. Proceedings of Petersburg Transport University, 2015, vol. 44, no. 3, pp. 77-84. In Rus.

23. Kopyrin V.A., Smirnov O.V., Portnyagin A.L., Khamitov R.N Influence of downhole compensator on voltage drop in elements of a production well electrical system. Bulletin of the Tomsk Polytechnic University. Geo Assets Engineering, 2018, vol. 329, no. 9, pp. 117-124. In Rus.

24. Novash I.V., Rumiantsev Yu.V. Three-phase transformer parameters calculation considering the core saturation for the MatlabSimulink transformer model. Energetika. Proceedings of CIS higher education institutions and power engineering associations, 2015, no. 9, pp. 12-24. In Rus.

25. Kosarev B.A., Fedorov V.K. Modeling of the power system with distributed generation. Omsk Scientific Bulletin, 2019, vol. 167, no. 5, pp. 64-71. In Rus.

26. Badalyan N.P., Kolesnik G.P., Andrianov D.P., Chebryakova Yu.S. Kabelnye $i$ vozdushnye linii elektroperedachi [Underground and overhead power supply lines]. Vladimir, VlGU Publ., 2019. 260 p.
27. Fayziyev M.M., Kurbonov N.A., Imomnazarov A.B., Bobonazarov B.S., Bekishev A.E. Simulation run asynchronous motor in MatLab. Bulletin of Science and Education, 2017, vol. 1, no. 5(29), pp. 10-14. In Rus.

28. Bulatov Yu.N., Kryukov A.V., Khyng Ch.Z. Automatic regulators for installations of distributed generation. Systems. Methods. Technologies, 2014, vol. 23, no. 3, pp. 108-116. In Rus.

29. Venikov V.A. Perekhodnye elektromekhanicheskie protsessy $v$ elektricheskikh sistemakh [Transient processes in electrical power systems]. Moscow, Vysshaya shkola Publ., 1985. 536 p.

30. Gaysarov R.V. Rezhimy raboty elektrooborudovaniya elektricheskikh stantsiy i podstantsiy: Chast 1. Rezhimy raboty sinkhronnykh generatorov $i$ kompensatorov [Operating modes of electrical equipment of electric power plants and substations: Part 1. Operating modes of synchronous generators and condensers]. Chelyabinsk, YUUrGU Publ., 2005. 42 p.

31. Ilyushin P.V., Kulikov A.L. Avtomatika upravleniya normalnymi $i$ avariynymi rezhimami energorayonov $s$ raspredelennoy generatsiyey [Automatic controls of typical and post-accident operation of power districts with distributed generation]. N. Novgorod, NIU RANKhiGS Publ., 2019. 364 p.

32. Vahdati P.M., Kazemi A. Bifurcations and chaos in nonlinear dynamics of power systems. 24th Iranian Conference on Electrical Engineering (ICEE). Shiraz, 2016. pp. 1706-1711.

33. GOST 32144-2013. Elektricheskaya energiya. Sovmestimost tekhnicheskikh sredstv elektromagnitnaya. Normy kachestva elektricheskoy energii v sistemakh elektrosnabzheniya obshchego naznacheniya. Mezhoosudarstvenny standart [State Standard 32144-2013. Electric Energy. Electromagnetic compatibility of technical equipment. Quality standards of electrical power of general-duty power supply systems. Interstate standard]. Moscow, Standartinform Publ., 2014. 20 p.

34. GOST R 50034-92. Sovmestimost tekhnicheskikh sredstv elektromagnitnaya. Dvigateli asinkhronnye napryazheniem do $1000 \mathrm{~V}$. Normy $i$ metody ispytaniy na ustoychivost $k$ elektromagnitnym pomekham [State Standard R 50034-92. Electromagnetic compatibility of technical equipment. Asynchronous motor with voltage up to $1000 \mathrm{~V}$. Testing Programme and Procedure for immunity to a disturbance]. Moscow, Standartinform Publ., 2004. 8 p.

35. Rysev P.V., Rysev D.V., Fedorov V.K., Shulga K.S., Pruss S.Y. Identifying and modeling chaotic modes in electrical power systems. Dynamics of Systems, Mechanisms and Machines. Proc. of the eleventh International IEEE scientific and technical conference. Omsk, OmGTU Publ., 2017. Vol. 5, no. 3, pp. 101-107.

36. Wolf A., Swift J.B., Swinney H.L., Vastano J.A. Determining Lyapunov exponents from a time series. Physica D: Nonlinear Phenomena, 1985, vol. 16, no. 3, pp. 285-317.

37. Mork B.A. Understanding and dealing with ferroresonance. Minnesota Power Systems Conference. St. Paul, 2006. pp. 1-10.

Received: 5 August 2020.

\section{Information about the authors}

Boris A. Kosarev, engineer, Omsk State Technical University.

Vladimir K. Fedorov, Dr. Sc., professor, Omsk State Technical University.

Rustam N. Khamitov, Dr. Sc., professor, Omsk State Technical University; professor, Tyumen Industrial University. 